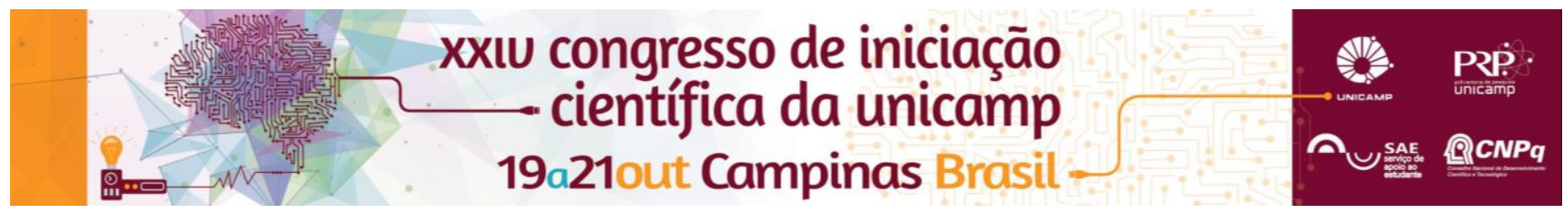

\title{
The discovery of natural products with antifungal activities through microorganism competition
}

\section{Cristiane I. Wassano*, Célio Fernando Figueiredo Angolini, Marcos Nogueira Eberlin, Taícia P. Fill}

\begin{abstract}
The co-culture between Penicillium digitatum and Penicillium italicum (both pathogens of citrus fruits) provides competitive and mutualistic forces that induce the synthesis of chemically different metabolites, as a defense mechanism, which can inhibit the growth of neighboring species.
\end{abstract}

Key words:

Co-culture, natural products, antifungals

\section{Introduction}

Since Brazil is the largest producer and exporter of oranges in the world, the demand for techniques aiming quality control is indispensable considering the losses caused by post-harvest fungal diseases ${ }^{1}$. The main causes of orange rot are the fungi Penicillium digitatum and Penicillium italicum (the two major citrus pathogens). This research focuses on the interactions between these two fungi through mixture cultures. The microbial competition for limited space and nutrients is a major ecological force that could activate silent gene clusters leading to the production of bioactive secondary metabolites, also called natural products, which are not directly involved in growth, development or reproduction of the producing organism, but provide advantages in many aspects to maintain its survival ${ }^{2}$.

Co-cultures are highly relevant for drug discovery and they allow not only the identification of new compounds, but also to investigate chemical events that govern interactions between microorganisms ${ }^{3}$.

\section{Results and Discussion}

To investigate the effect of mixed cultivation on secondary metabolism, it was carried out single and coculture experiments with Penicillium digitatum and Penicillium italicum, in PDA( Potato Dextrose Agar) solid media. In the co-culture experiment a growth inhibition between the two fungi was identified as seen in Figure 1, suggesting the production of antifungal defense metabolites.

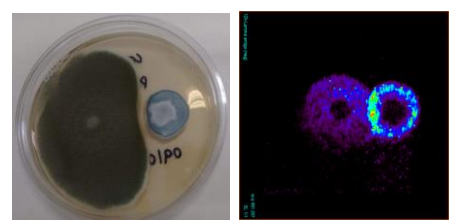

Figure 1. A) Co-culture between Penicillium digitatum and Penicillium italicum. B) Mass Spectrometry Imaging (MSI) of the co-culture of Penicillium digitatum and Penicillium italicum.

In order to evaluate metabolite production, the coculture interface was extracted with dichloromethane, ethyl acetate and methanol. The extracts were analyzed by HPLC/MS and Mass Spectrometry Imaging (MSI).

Interactions among neighboring fungi exhibited significant chemical changes relative to their respective DOI: 10.19146/pibic-2016-51347 single cultures. Figure 1B shows mass spectrometry imaging (MSI) of the co-culture. It is possible to visualize the spatial distribution of the chemical compounds produced by the fungi in a competitive environment and understand how the compounds are involved in the chemical interaction guiding us to antifungal natural products discovery.

In order to evaluate the fungal extracts concerning antifungal activity, a biological assay was performed in a 96-well plate, by adding $50 \mu \mathrm{L}$ of liquid medium PD (Potato Dextrose), $30 \mu \mathrm{l}$ milli-Q water and $20 \mu \mathrm{l}$ of Penicillium digitatum spore suspension in each well, followed by addition of extract of co-cultivation. The growth inhibition of Penicillium digitatum caused by secondary metabolites synthesized in co-culture is shown in Figure 3, columns 4,5 and 6 .

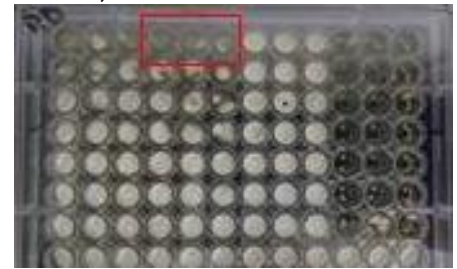

Figure 3. Antifungal activity assay with co-colture extracts.

\section{Conclusions}

The competition between microorganisms is a powerful method for the discovery of new bioactive natural products. In a competitive environment, Penicillium italicum produces chemically different substances that inhibits the growth of Penicillium digitatum, species that dominates the culture medium when cultivated alone. The antifungal metabolites induced are now under investigation in our laboratory.

\section{Acknowledgement}

This work was supported by FAPESP $(2015 / 10384$ 6). We also thank "Fundo de Apoio ao Ensino, Pesquisa e Extensão" (FAEPEX).

${ }^{1}$ Fischer, I. H.; Lourenço, S. A.; Amorim, L. Doenças pós colheita em citros e caracterização da população fúngica ambiental no mercado atacadista de São Paulo. Trop. Plant Pathol. v. 33, p. 219-226, 2008.

${ }^{2}$ Bertrand S, et al,Metabolite induction viamicroorganism co-culture: A potential way to enhance chemical diversity for drug discovery, Biotechnol Adv (2014).

${ }^{3}$ Chagas, F. O.; Dias, L. G.; Pupo, M. T. A mixed culture of endophytic fungi increases production of antifungal polyketides. Journal of Chemical Ecology, v. 39, n. 10, p. 1335-1342, 2013 